\title{
MHD PbLi Experiments in MaPLE Loop at UCLA
}

\author{
C. Courtessole, S. Smolentsev, T. Sketchley, M. Abdou \\ Fusion Science \& Technology Center, University of California, Los Angeles, USA
}

\begin{abstract}
Experiments on magnetohydrodynamic (MHD) flows are critical to understanding complex flow phenomena in ducts of liquid metal blankets, in particular those that utilize eutectic alloy lead-lithium as breeder/coolant, such as self-cooled, dual-coolant and helium-cooled lead-lithium blanket concepts. The primary goal of MHD experiments at UCLA using the liquid metal flow facility called MaPLE (Magnetohydrodynamic PbLi Experiment) is to address important MHD effects, heat transfer and flow materials interactions in blanket-relevant conditions. The paper overviews the one-of-a-kind MaPLE loop at UCLA and presents recent experimental activities, including the development and testing of high-temperature PbLi flow diagnostics and experiments that have been performed since the first loop operation in 2011. We also discuss MaPLE upgrades, which need to be done to substantially expand the experimental capabilities towards a new class of MHD flow phenomena that includes buoyancy effects.
\end{abstract}

Keywords: Magnetohydrodynamics (MHD) experiment, liquid metal loop, lead-lithium (PbLi), flow diagnostics.

\section{Introduction}

Eutectic alloy lead-lithium $(\mathrm{PbLi})$ is a very attractive liquid metal (LM) material as breeder/coolant for fusion applications given its insensitivity to radiation damage and ability for in-situ control of the breeding rate. However, the Lorentz forces induced by the motion of the LM in a strong plasma-confining magnetic field lead to magnetohydrodynamic (MHD) effects that can tremendously increase the pressure drop and strongly affect the flow distribution as well as heat and mass transfer processes. Since PbLi was first proposed as a breeder material in the early 1980's, many experimental and theoretical studies on transport phenomena in $\mathrm{PbLi}$ flows in fusion-relevant conditions have been carried out, yet many fundamental and practical aspects still need to be investigated. In particular, the performance of insulation techniques such as a flow channel insert (FCI) employed, for example, in the dual-coolant lead-lithium (DCLL) blanket [1] still need to be addressed along with the effect of buoyancy forces on MHD flows and the influence of the magnetic field on the corrosion of structural and functional materials as well as tritium transport. R\&D is also required to develop new diagnostic tools suited for high-temperature PbLi.

To address these issues, a MHD PbLi loop called MaPLE (Magnetohydrodynamic PbLi Experiment) was constructed at University of California, Los Angeles in 2011 [2]. Since then, the loop was upgraded several times. First, the loop was mounted on a wheeled platform so that it could be moved in and out of the gap of the electromagnet. Then, the PbLi inventory was increased from $70 \mathrm{~kg}$ to about $200 \mathrm{~kg}$. Finally, a purification unit was connected to the loop to control the oxygen level in the cover argon gas in the melting tank.

This paper presents the most recent upgrades and the status of the MaPLE facility. It overviews the progress on developing and testing of high-temperature $\mathrm{PbLi}$ instrumentation and summarizes the most important MHD experiments that have been carried out in the facility since 2011. The last section describes the nearterm plans on facility upgrades to substantially increase the experimental capabilities towards a new class of MHD flow phenomena that includes buoyancy effects.

\section{Recent upgrade of the MaPLE facility and status}

The current view of MaPLE is shown in Fig. 1. The key component of the facility is a $15-\mathrm{cm}$ gap electromagnet in which a PbLi loop can be inserted. The magnet, made of ten water-cooled copper coils, can produce a uniform magnetic field up to $1.8 \mathrm{~T}$ within a $80-\mathrm{cm}($ axial $) \times 15-\mathrm{cm}($ horizontal $) \times 15-\mathrm{cm}($ vertical $)$ region.

To facilitate the maintenance of the facility, the whole loop sits on a cart so that it can be moved in the magnet gap during experimental campaigns and pulled out for the rest of the time. A new melting/draining tank of about $300-\mathrm{kg}$ capacity has been added to make standard loop operations easier and faster. All the loop components, namely the reservoir, the 1-inch $(25.4 \mathrm{~mm})$ pipes, the electromagnetic (EM) flowmeter, the test section, and the draining tank are made of stainless steel AISI 304; only the EM pump channel is made of another grade of stainless steel (AISI 316). The EM conduction pump, manufactured by Creative Engineers, Inc., USA, develops a maximum pressure head of $2.0 \times 10^{5} \mathrm{~Pa}$ and can be operated up to a temperature of $530{ }^{\circ} \mathrm{C}$. However, to prevent the corrosion of the pipes, the nominal temperature of the liquid metal is limited to $350{ }^{\circ} \mathrm{C}$. The whole loop is thermally insulated, and up to $10 \mathrm{~kW}$ of power can be provided through the heaters to keep the liquid metal above its melting point $\left(235^{\circ} \mathrm{C}\right)$. To avoid oxidation of the liquid metal, a glove box, which sits on the top of the reservoir, is connected to a purification unit that keep the oxygen concentration under $5 \mathrm{ppm}$ in the argon gas atmosphere above the free surface of the liquid metal.

author's email: cyril@fusion.ucla.edu 


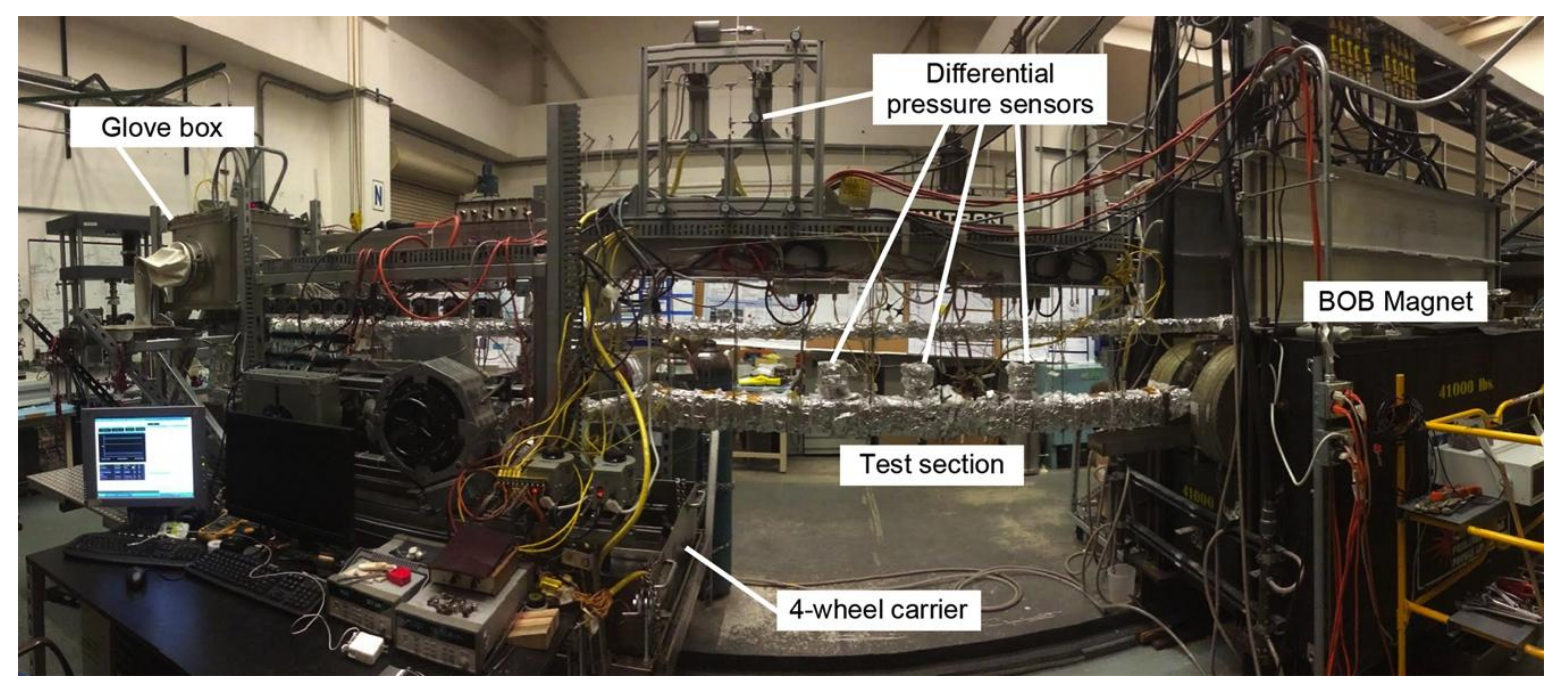

Fig. 1. Panoramic photo of the MaPLE facility at UCLA used for dynamic testing of the FCI sample.

Recently, the facility has been upgraded to allow for the 190-cm long test section to be replaced easily. Instead of being welded on the loop, the test article is mounted using stainless steel flanges (Class 150) and graphite gaskets. A total of three different rectangular ducts and one circular 1-inch pipe have been fabricated to be used in experiments. Depending on the test section employed, the PbLi inventory varies between $180 \mathrm{~kg}$ and $300 \mathrm{~kg}$, and the maximum flowrate is between $15 \mathrm{l} / \mathrm{min}$ and $35 \mathrm{l} / \mathrm{min}$ under a magnetic field of $1.5 \mathrm{~T}$.

\section{Diagnostic and flow control}

Measurement in molten $\mathrm{PbLi}$ is exceptionally challenging because of the aggressive nature of $\mathrm{PbLi}$ and its high temperature. Therefore, commercial flow diagnostics are very limited. That is why a significant effort has been taken to improve existing and to develop new diagnostics tools for $\mathrm{PbLi}$ flows.

\subsection{Temperature measurements}

Temperature is probably the easiest physical quantity to measure. Thermocouples are cheap, widely available and can be commonly found. They can be protected by a sheath made of various materials (the most common sheath material being stainless steel or Inconel). In the MaPLE facility, most of the thermocouples used are type- $K$ thermocouples and are installed directly on the

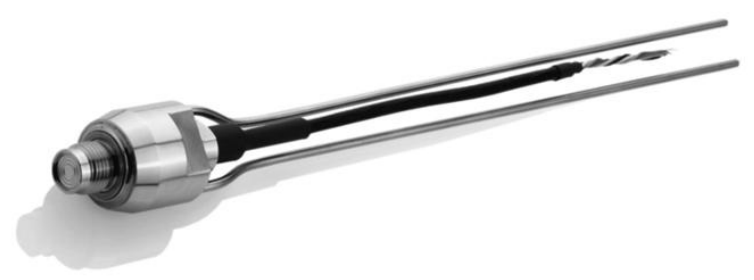

Fig. 2. High-temperature pressure transducer tested on MaPLE loop (Series 13, Keller AG). pipes of the loop. Three different types of thermocouples $(\mathrm{E}, \mathrm{K}$ and $\mathrm{N})$ protected by a stainless steel sheath (AISI 304) have also been successfully tested to directly measure the temperature in the flowing $\mathrm{PbLi}$ between $250{ }^{\circ} \mathrm{C}$ and $350^{\circ} \mathrm{C}$, demonstrating very consistent results since all three readings were accurate to the uncertainty of the thermocouple (i.e. $\pm 1^{\circ} \mathrm{C}$ ).

\subsection{Pressure measurements}

In addition to developing an indirect differential pressure measurement technique as described in [3], a commercial absolute pressure transducer manufactured by Keller AG, Switzerland, has been tested (Fig. 2).

Designed to operate up to $350{ }^{\circ} \mathrm{C}$ between 0 and 10 bars, the commercial pressure transducer (Series 13) integrates four stain gauge resistors located in the watercooled section of an oil-filled body. The hightemperature oil transmits the pressure from the stainless steel membrane (AISI 316) in contact with the hot liquid metal to the sensor. Before installing these pressure transducers on the MaPLE loop, static tests were performed to calibrate them and to verify their compatibility with liquid PbLi. The tests confirmed that the instrument is suitable for use with $\mathrm{PbLi}$ as long as the temperature of the liquid metal is less than $350{ }^{\circ} \mathrm{C}$. The transducer shows no problem in a $1.5 \mathrm{~T}$ magnetic field.

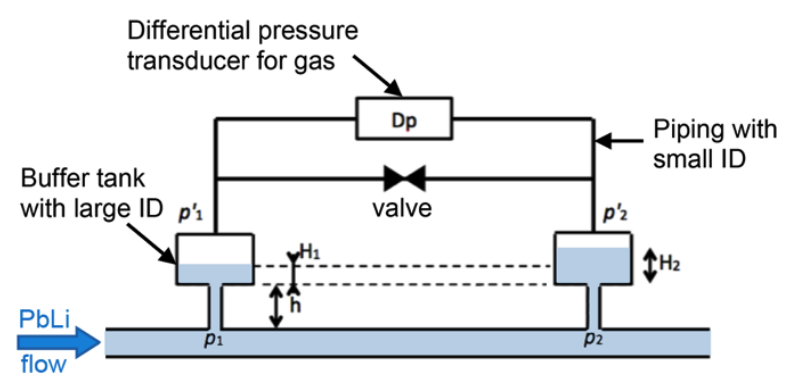

Fig. 3. Sketch of the indirect differential pressure method employed. 


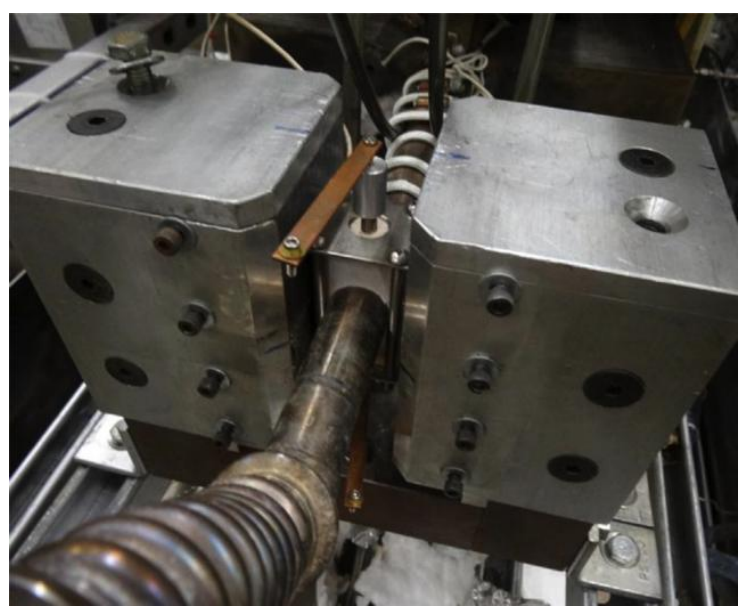

Fig. 4. EM flowmeter made of two permanent magnets attached to an iron magnetic yoke.

UCLA also develops an indirect differential pressure measurement technique where a normal gas-pressure transducer (DP-15 from Validyne Engineer Corp., USA) is employed as shown in Fig. 3. This technique relies on the isolation of the temperature-limited diaphragm of the transducer from liquid $\mathrm{PbLi}$ using argon gas. Two identical buffer tanks $(\mathrm{D}=73.2 \mathrm{~mm}$ and $\mathrm{h}=47 \mathrm{~mm})$ made of stainless steel are installed on the test-section. They are partially filled with argon and connected to the differential pressure transducer by very small ID $(1.4 \mathrm{~mm}$ and $0.76 \mathrm{~mm})$ stainless steel pipes so that the volume of these pipes remains negligible relative to the volume of gas in the buffer can.

\subsection{Flow-rate measurements}

Flow-rate measurements are performed using a homemade EM flowmeter. The principle of this socalled Faraday flowmeter is to apply an external transverse DC magnetic field to induce currents in the flowing liquid metal; the measurement of the potential difference in the direction orthogonal to the magnetic field and to the flow yields the flowrate. According to Shercliff [4], the sensitivity $S$ of an EM flowmeter made of circular pipe, where contact resistances are neglected, is given by

$$
S=\frac{V}{2 R B_{0} U_{0}}=\frac{2 r^{2}}{\left(r^{2}+R^{2}\right)+\sigma / \sigma_{w}\left(R^{2}-r^{2}\right)}
$$

where $V$ is the electric potential difference measured between the electrodes, $B_{0}$ the magnetic field, $U_{0}$ the mean flow velocity, $r$ and $R$ the inner and outer radius of the pipe, and $\sigma$ and $\sigma_{w}$ the electric conductivity of the fluid and of the pipe respectively.

In the effort to improve upon the EM flowmeter that traditionally employs two permanent magnets installed on an iron yoke that concentrates the magnetic flux

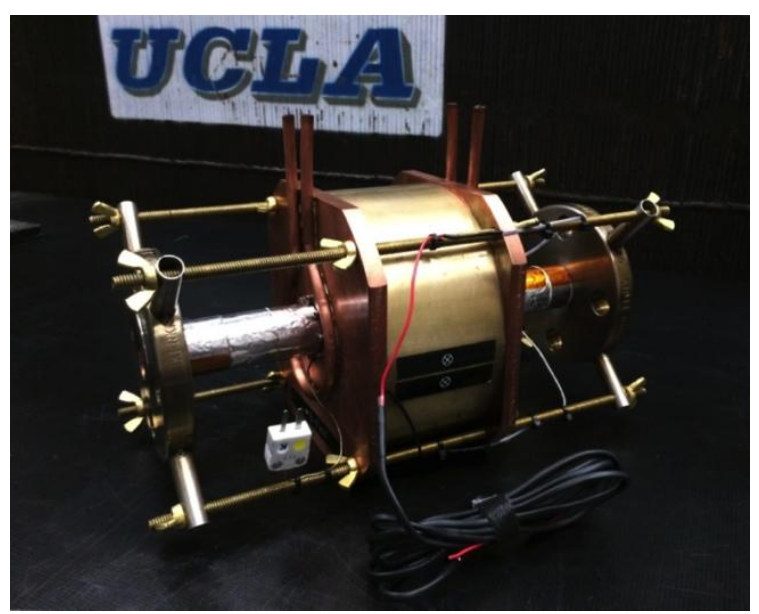

Fig. 5. Improved EM flowmeter based on a Halbach cylinder.

(Fig. 4), we developed a new EM flowmeter based on a specific arrangement of eight $\mathrm{NdFeB}$ permanent magnets known as a Halbach cylinder (Fig. 5) [5]. The principle is still the same; two stainless steel electrodes are welded on a 25.4-mm OD stainless steel pipe (22.2 mm ID) of the flowmeter to measure the induced voltage, but unlike the flowmeters made of two permanents magnets that produce a non-uniform and relatively weak magnetic fields, the Halbach cylinder employed here does not suffer these drawbacks and provides a uniform magnetic field in its cavity of $0.85 \mathrm{~T}$.

\subsection{Electric potential measurements}

Electric potential measurements on the conductive wall of the test article are also commonly performed through spot-welded stainless pins on the test section.

\subsection{Experimental studies in MaPLE}

\subsection{MHD flow in a fringing magnetic field}

MaPLE was first used to investigate the effect of a fringing (non-uniform) magnetic field on $\mathrm{PbLi}$ flows in electrically conductive ducts and to establish a reliable approach for measuring the pressure drop in a hightemperature liquid metal [3]. In this experiment, the test section was made of a 1 -inch stainless steel (AISI 304) pipe $(25.4 \mathrm{~mm}$ OD and $22.1 \mathrm{~mm} \mathrm{ID)}$ and the flow diagnostics were limited to pressure drop measurement using the indirect differential method described in section 3.2. Only flow entering in the fringing field was experimentally investigated and the obtained results were compared to numerical 3D simulations performed with HIMAG [6]. Data were taken at Hartmann number up to 320 and Reynolds number up to $5 \times 10^{4}$.

This study demonstrated the reliability of the pressure system as verified with a simple formula for MHD pressure drop proposed by Miyazaki et al. [7]. 

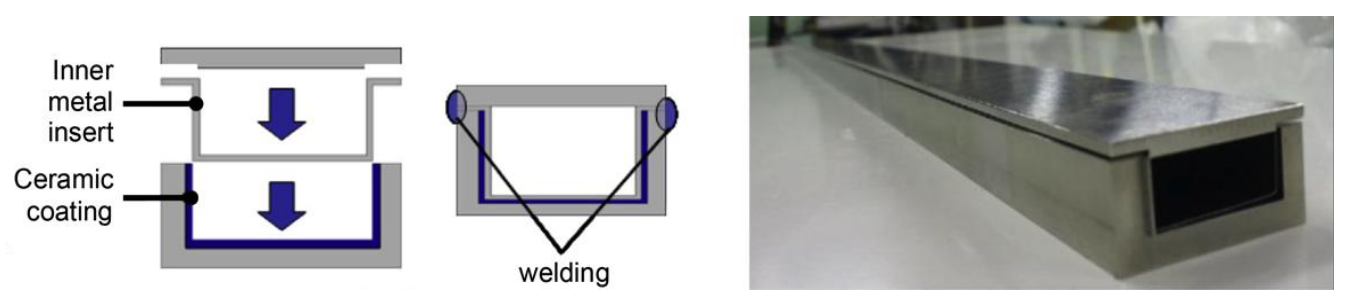

Fig. 6. Multi-layered duct fabrication process (left) and photograph (right).

\subsection{MHD pressure drop reduction experiments}

High MHD pressure drop is one of the feasibility issues in almost all LM blankets. To reduce the MHD pressure drop several approaches have been proposed. Malang first recommended an alumina-steel-alumina FCI as electrical insulator. Later, silicon carbide (SiC) FCI was proposed to replace the sandwich insert as both electrical ant thermal insulator to substantially increase the exit temperature of the liquid metal to $700{ }^{\circ} \mathrm{C}$ [8]. Other authors suggested laminated walls [9]. Hashizume adapted this concept to a self-cooled lead-lithium blanket where only three out of the four walls are insulated with a thin ceramic layer [10].

\subsubsection{Three-wall multi-layered duct experiment}

In the framework of the US-Japan "TITAN" program [11], a three-sided multi-layered duct was fabricated at Tohoku University, Japan, and tested in the MaPLE facility. Three inner walls of the $80-\mathrm{cm}$ long stainless steel (AISI 316) test channel were painted with a 0.05 to $0.15 \mathrm{~mm}$ silica layer before installing another $0.1 \mathrm{~mm}$ thick layer of stainless steel inside the channel and closing the channel with the fourth $3-\mathrm{mm}$ thick wall made of stainless steel only (Fig. 6). All the parts were welded together and the resulting test channel inner height was $8 \mathrm{~mm}$ with a $20-\mathrm{mm}$ width and the thickness of each layered wall was $5 \mathrm{~mm}$.

The test article was installed on the loop and the pressure drop was measured over the duct section where the flow was expected to be fully developed. The magnetic field was varied from 0 to $1.8 \mathrm{~T}$, the mean velocity from 0 to $2.0 \mathrm{~m} / \mathrm{s}$ corresponding to a Reynolds number up to $1.1 \times 10^{5}$ and Hartmann number up to 380 . The comparison of experimental data with numerical simulations for a three-wall multi-layered duct and for a non-insulated duct demonstrated an important pressure drop reduction of about a factor of 10 . More details of the experimental procedure are reported on [12].

\subsection{2 $\mathrm{SiC}$ foam-based FCI experiment}

More recently, we performed a feasibility study of a SiCfoam based FCI manufactured by Ultramet, USA, lasting 6500 hours. The tested $30-\mathrm{cm}$ long FCI sample was made of low-density, porous ( $85 \%) \mathrm{SiC}$ foam filled with aerogel $(8 \mathrm{~mm})$, coated with a $1-\mathrm{mm}$ thick SiC CVD layer (chemical vapor deposition) [13]. Its initial weight was $780 \pm 1 \mathrm{~g}$. The SiC FCI was inserted into a $90-\mathrm{mm} \times$ $90-\mathrm{mm} \times 1848-\mathrm{mm}$ host stainless steel duct with 3-mm walls fabricated at UCLA as shown in Fig. 7. Two restraining pins are used to prevent the FCI from being dislodged by the flow, and eight pins ensure it is centered in the host duct, leaving a 2-mm gap between the FCI and the walls of the host duct. Three pressure taps are welded on the duct, $34 \mathrm{~cm}$ and $4 \mathrm{~cm}$ upstream and $4 \mathrm{~cm}$ downstream of the FCI, to connect the indirect differential pressure measurement system.

Pressure drops were measured over a $30-\mathrm{cm}$ section of the bare duct and over a $38-\mathrm{cm}$ section that included the FCI, first with no magnetic field and then at three different magnetic field strengths $(0.5,1.0$ and $1.5 \mathrm{~T})$ and at various flowrates up to $38 \mathrm{l} / \mathrm{min}$ (corresponding to a mean velocity in the bare duct of $9.0 \mathrm{~cm} / \mathrm{s}$ ). During the experiments, the liquid metal temperature was kept at $300 \pm 5{ }^{\circ} \mathrm{C}$. The maximum non-dimensional numbers associated with this experiment are $\mathrm{Re}=16600$ and $\mathrm{Ha}=1200$.

Surprisingly, the comparison between the pressure drop measured over the FCI segment with data obtained for the bare duct presents an unexpected trend; whatever the magnetic field strength is, the pressure drop is higher over the FCI than that for the PbLi flow in the bare duct (Fig. 8). Thus, the pressure drop reduction factor, or Rfactor, which is defined as the ratio of the pressure drop due to MHD effects without an FCI relative to that with a FCI, is always lower than unity. Not only these results are opposite to our expectations, but also they are in

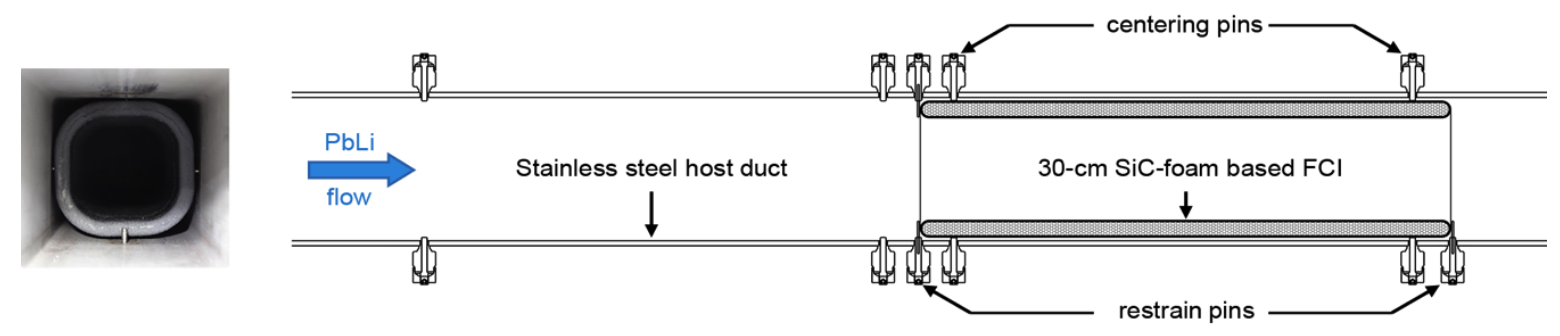

Fig. 7. Photograph (left) and sketch of the flow-channel insert inside the host duct. 


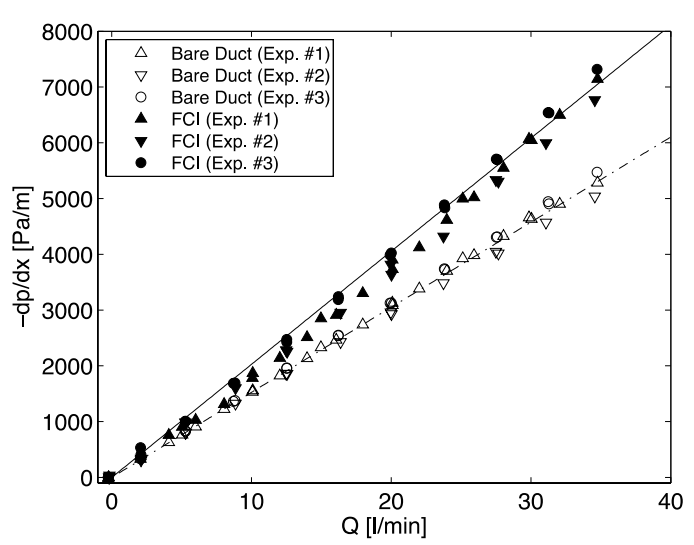

Fig. 8. Comparison of the MHD pressure drops in the bare duct and over the FCI at $\mathrm{B}=1.03 \mathrm{~T}$.

contradiction with the full 3D computations performed as pre-experimental analysis using the HIMAG code at electric conductivity of the $\mathrm{SiC} \mathrm{FCI}$ of $5 \mathrm{~S} / \mathrm{m}$. The predicted pressure drop reduction factors are in the range of 1.0 to 1.5 depending on the magnetic field; they are compared with experimental values in Table 1

Degradation of insulating properties of the FCI caused by liquid metal ingress into the porous core of the material explains the underperformance of the $\mathrm{SiC}$ sample tested. After the experimental campaign, the loop was drained and the FCI extracted from the duct. Although no visible cracks were noticed on the protective CVD layer that could have shown some evidence of an entrance path for the liquid metal, the FCI was weighed at $3022 \pm 1 \mathrm{~g}$, thus gaining $2242 \mathrm{~g}$ compared to its initial mass of $780 \mathrm{~g}$, confirming PbLi filled about $45 \%$ of the pores of the foam. Post-mortem micrograph analyses were performed to corroborate this massive ingress. The FCI sample was cut into a few $1-\mathrm{cm}$ thick slices that have been inspected using an optical microscope. Wherever the samples were taken from the FCI (inlet, middle or outlet section, on the Hartmann wall or on the sidewall), all the micrographs clearly demonstrate the presence of $\mathrm{PbLi}$ in the pores of the core structure of the insert as shown in Fig. 9.

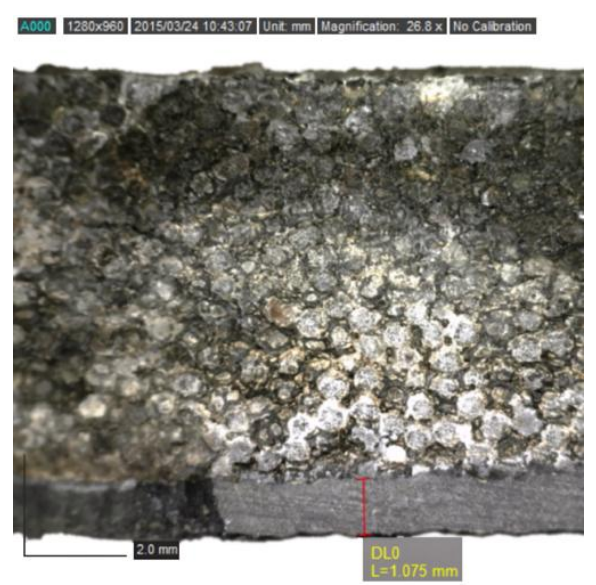

Fig. 9. FCI micrograph taken in the middle of the FCI sample, on the sidewall.
Table 1. Comparison of measured and simulated R-factors.

\begin{tabular}{ccc}
\hline $\mathrm{B}[\mathrm{T}]$ & Numeric & Experiment \\
\hline 0.5 & 1.05 & 0.73 \\
1.0 & 1.37 & 0.75 \\
1.5 & 1.52 & 0.78 \\
\hline
\end{tabular}

\subsection{Ongoing experiment: Heat transfer in MHF flows}

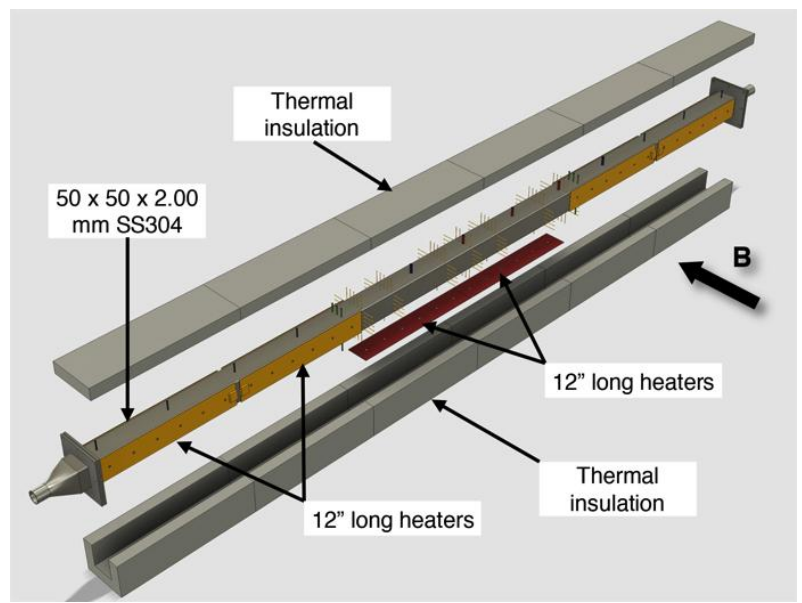

Fig. 10. Sketch of the test article used for the heat transfer experiment.

Because it is of utmost importance to understand the effect of buoyancy and control heat transfer in a liquid metal blanket, we have designed an experiment that aims at measuring the heat transfer coefficient in PbLi MHD flows depending on different flow regimes. As shown in Fig. 10, a new 50-mm $\times 50-\mathrm{mm} \times 1890-\mathrm{mm}$ test article with $2 \mathrm{~mm}$ walls has been fabricated to be installed on MaPLE. Mica Thermofoil ${ }^{\mathrm{TM}}$ heaters (Minco, USA) are used to heat the test article and provide up to $10 \mathrm{~W} / \mathrm{cm}^{2}$ very uniform heat flux density on the bottom wall of the test section. A set of eight heaters (in yellow on Fig. 10), located upstream and downstream of the $60-\mathrm{cm}$ uniform heat flux region on the Hartmann walls, serves to heat the duct during the filling of the loop and between experiments. During the experiments, these heaters are turned off and only the two heaters clamped on the bottom wall are energized. To minimize the heat loss, the test article is enclosed in a 1-inch $(25.4 \mathrm{~mm})$ thick refractory material (Zircal-18 from ZIRCAR Refractory Composites, Inc., USA) that has a very low thermal conductivity $\left(0.07 \mathrm{~W} /(\mathrm{m} . \mathrm{K})\right.$ at $\left.200{ }^{\circ} \mathrm{C}\right)$. Several ports have been welded on the rectangular duct to introduce instrumentation into flowing $\mathrm{PbLi}$. In addition to temperature sensors and electric potential probe that are installed on the duct, two temperature integrators (made of an array of 16 thermocouples) are inserted into the liquid metal to measure the mean temperature over the cross section of the duct along with movable probes that are used to measure the temperature distribution inside the liquid metal. LEVI probes are used to measure the local velocity in the liquid metal. 


\section{Near-term upgrade of the facility and experiments}

The Maple loop is currently limited to horizontal MHD flows. However, in a fusion reactor, blanket modules have different orientations with respect to gravity. Depending on the orientation of the flow, one can expect different flow regimes, where buoyancy can dominate over inertia, viscous and even electromagnetic forces. Therefore, to design a liquid metal blanket, it is critical to study MHD flows at different orientations with respect to gravity.

An exploratory study has been initiated at UCLA to upgrade the facility to various flow orientations. In this context, we consider different design scenarios where the magnet and the test article could be tilted to any inclination angle from 0 to $90^{\circ}$ to allow for a whole new class of experiments on MHD mixed convection.

In parallel, we are currently planning to increase the temperature and the surface heat flux that can be imposed on a wall of the test article to simulate prototypical fusion conditions and explore a larger range of experimental parameters.

\section{Conclusion}

This paper overviews recent experimental activities carried out in the MaPLE facility at UCLA, which include (1) improvement or development of instrumentation for high-temperature $\mathrm{PbLi}$ and (2) studies of the influence of a magnetic field on $\mathrm{PbLi}$ flows.

So far, effort has mainly been put into testing insulation techniques to reduce the MHD pressure drop. If the multi-layered proof-of-concept experiment demonstrated good results, the feasibility tests for $\mathrm{SiC}$ FCI clearly underscore the need for further improvements in manufacturing SiC FCI to guarantee no $\mathrm{PbLi}$ ingress.

In addition to the continuation of these two activities, near-term experiments have been designed to focus on heat transfer in MHD PbLi flows. We also plan major upgrades of the facility by developing a magnet tilting system to study the influence of buoyancy on forced MHD flows, or so-called mixed convection flows, at different flow orientations with respect to gravity.

\section{Acknowledgments}

This work is supported by the U.S. Department of Energy, Office of Science, Office of Fusion Energy Sciences, under Award Number DE-FG02-86ER52123.

\section{References}

[1] S. Smolentsev, N. B. Morley, M. A. Abdou, and S. Malang, Dual-coolant lead-lithium (DCLL) blanket status and R\&D needs, Fusion Engineering and Design (2015) (In press).
[2] S. Smolentsev, F-C. Li, N. Morley, Y. Ueki, M. Abdou and T. Sketchley, Construction and initial operation of MHD PbLi facility at UCLA, Fusion Engineering and Design 88 (2013) 317-326.

[3] F-C. Li, D. Sutevski, S. Smolentsev and M. Abdou, Experimental and numerical studies of pressure drop in $\mathrm{PbLi}$ flows in a circular duct under non-uniform transverse magnetic field, Fusion Engineering and Design 88 (2013) 3060-3071.

[4] J.A. Shercliff, The Theory of Electromagnetic FlowMeasurement, Cambridge University Press, London, 1962.

[5] K. Halbach, Design of permanent multipole magnets with oriented rare earth cobalt material, Nuclear Instruments and Methods 169 (1980) 1-10

[6] S. Smolentsev, N.B. Morley, M. Abdou, R. Munipalli and R. Moreau, Current approaches to modeling MHD flows in the dual coolant lead lithium blanket, Magnetohydrodynamics 42 (2006) 225-236.

[7] K. Miyazaki, S. Inoue, N. Yamaoka, T. Horiba and K. Yokomizo, Magnetohydrodynamic pressure drop of lithium flow in rectangular ducts, Fusion Technology 10 (1986) 830-836

[8] S. Malang, M. Tillack, C.P.C. Wong, N. Morley and S. Smolentsev, Development of the lead lithium (DCLL) blanket concept, Fusion Science and Technology 60 (2011) 249-256

[9] D.L. Smith, C.C. Baker, D.K. Sze, G.D. Morgan, M.A. Abdou, S.J. Piet et al., Overview of the blanket comparison and selection study, Fusion Technology 8 (1985) 10-44.

[10] H. Hashizume, Numerical and experimental research to solve MHD problem in liquid blanket system, Fusion Engineering and Design 81 (2006) 1431-1438.

[11] S. Smolentsev, T. Kunugi, K. Messadek, T. Yokomine, J. Young et al., Status of "TITAN" Task 1-3 "Flow Control and Thermofluid Modeling", Fusion Engineering and Design 87 (2012) 777-781.

[12] M. Aoyagi, Y. Inage, S. Ito, S. Ebara, Y. Ueki, K. Yuki et al., Verification test of a three-surface-multi-layered channel to reduce MHD pressure drop, Proceedings of the $8^{\text {th }}$ JapanKorea Symposium on Nuclear Thermal Hydraulics and Safety (NTHAS8), 9-12 December 2012, Beppu, Japan (2012).

[13] S. Sharafat, B. Williams et al., Development status of a SiC-foam based flow channel insert for a U.S.-ITER DCLL TBM, Fusion Science and Technology 56 (2009) 883-89. 